de ce còté des Alpes que M. Aristide Bergès, en 1869 , ¿ I.ancey, M. Charles Chevrant, en r 888 , à Revel, et M. Bravel, en $18 g h$, à Chapareillan, onl fait les permières installations de hautes chutes.

Après ces pionniers, admirablement sccondés et aidés par les constructeurs de Grenoble, les Bouchayer et Viallet, les Joya, les Brenier-Neyret, les Bouvier, clc..., la voie était tracée, les principes posés et les éléments de calcul appuyés sur des faits écartant toute incertitude.

Nous pouvons applaudir aux succès des ingénieurs qui ont aménagé les chutes de l'Adamello, parce que nous pouvons constaler avec satisfaction que les ingénieurs et les conslrucleur's francais, en réalisant la chute d'Orlu $(936 \mathrm{~m}$.) dans l'Ariège et celle, presque verticale, de la Corbière $(736 \mathrm{~m}$.) en Savoie, ne se montrent point inférieurs en science, en ardace et en habileté à leurs confrères transalpins.

\section{LA NAVIGATION INTÉRIEURE EN SUISSE}

Notro excollent confrère le Bulletin technique de la Suisso. Romande publie une intéressante élude de M. A. ParssWrTrec sur les grands projets de navigation intérieure qui sont acluellement discutés en Suisse. Nous en exlrayons Jes renseignemenls suivants.

Le Conseil des Etats suisses est saisi du projet de législalion fédérale sur l'utilisation des forces hydrauliques, qui passera ensuile devant le Conseil national. Les Chambres vont donc prendre des dispositions lourdes de conséquences sur l'avenir de la navigalion sur les rivières suisses.

Nous avons signalé les grands travaux qui s'exéculent dans la Suisse romande, en particulicr le canal d'Entreroche, qui cst l'amorce de la grande voie navigable du Rhône au Rhin en pussant par les lacs jurassiens, l'Aar et le Rhin de Bâle a Constance. C'est cetle dernière partie de cette voie que les nouveaux projots de loi intéressent tout particulièrement

L'éludo de l'aménagement dos forces motrices du Rhin est cnlrée dans tune phase décisise. Tout récemment on a achevé la construction des usines d'Augst-Wylen, dont le barrage relicnt une hauteur' d'eau de $8 \mathrm{~m}$. environ et forme en amont un lac de $7 \mathrm{~km}$. de long. Le capital investi de $22 \mathrm{mil}$ lions en fait un ouvrage définitif. Et co n'est que grâce aux ffforts personnels des partisans de la voje d'eau que l'éclusc d'Augs a été conslruite en vue de la grande navigation. Le projet portait une écluse pour petits chalands et flottage, donl le coût eût été de 450 ooo francs. La grande écluse n’a pu êlre construite qu'ensuite d'apport de la différence de loo ooo francs, moitié par la Suisse, et moilié par le Grand Duché de Bade. Il est inadmissible que la Société, qui va s'enrichir en exploitant le bien commun, se soit fait payer près d'un demi-million pour respecter la navigabilité actuelle en ce point du Rhin. Le fait est d'autant plus singulier que la navigation internationale sur ce point était garantie par les traités (Vienno I8I5 ét traité entre la Suisse et le Grand Duché de Bade 1879 ). Sans l'énergie de l'ingénieur Gelpke, de Bâle, en particulier, c'est un million qu'il cût fallu débourser d'ici quelques années peut-être pour rendre au Rhin sa navigabilité pour les grands chalands.

L'expérience est malheureusement faite actuellement à Laufenburg. L'écluse insuffisante devra inévitablement être prolongée à grands frais plus tard, aux dépens du pays, el cecci pour remédier aux économies d'une société montée au copital de 40 millions. Et comme il y a en ce moment cing grands barrages sur le point de se construire sur le Rhin, la Suisse se demande avec inquićtude quelle atlitude la (anfédćration prend vis-à-vis des socićtés auxquelles elle siupprête à donner des concessions désormats. Fsl-elle arméc pour exiger le respect du bien public ? Le sera-t-elle apris lo vote de la loi en discussion? Rien n'est moins certain. En effet, l'article 6 intitulé " Protection de la navigation en général " dil que "les usines hydrauliques scront construites de façon à ne pas entraver la navigation dans la mesurc oì elle existe $n$. Or, le fleuve, dans l'étal actuel, avec ses rapidl:; ne permet pas la grande navigation intérieure, mais mo fois ces rapides apaisés el sa beauté, patrimoine national, en partie perdue, le fleuve sera de fait une série do lacs, el il serail facile de les mettro en communication suffisante cntro cux pour permetlre à la grande navigation existant en aval de profiter de ees nouvelles circonstances pour se prolonger en amont; el pénétrer au cour de la Suisse. Celle légitime compensation à la concession serail juste el admise sans difficullé par les requérants avanl la signature du contral. L'Autorité devrait seulement avoir te droit d'en dentander. l'exécution gratuile. Ce droil, c'est à la loi en discussion de l'octroyer sans limitation au pouvoir central, conseillé jart des techniciens experts. Si les Chambres n'y veillenl, a Suisse se trouvera dans quelques années coupéc de la mor par des écluses insuffisantes, el obligée de l'ansborter les grands chalands sur des bateaux proportionnés à ses petiles installations. A moins loutefois qu'dle ne préfere, à chaqua nouvelle construction, répéler lo paicment des indemnilí: réclamées par la Sociélé d'Augst, pour exéculer après signa t'ure du contral, ce gu'clle aurail fail gratis si on l'avail de mandé à temps. Il s'agissait pour elle d'une augmenlalion de moins de deux pour cent de son capilal de premier chlablissement. Les dividendes cux-mêmes ne s'en seraient pas ressentis.

La navigation inlérieure sur chalands suisses nculres peut seule la garantir contre l'embargo on cas de guerr. européenne. On se représente la diselte g'ui s'en suivrail, of ce que deviendrait son indépendance lorsque loules ses from. tières seraicnt fermées, el que les approvisionnements seraient impossibles. La navigation internationale, neutralisce" par les traités, est la vraje solution suisse de la question do: farines en particulier. Et le moyen de la methe sur pied i bref délai est bien de lui assurer d'ores et déjà les outils dont elle a besoin, sans lui occasionner par la suite des fraits qui mellraient sa justification financière en question.

Gette justification ne saurait en effet tarder. La premic̀r année d'exploitation, xgoo, indiquait un trafic Strasbourg. Bâle de 300 lonnes. La dixième année, rgog, on notait 4o8 89 , et l'année courante, $\mathrm{Ig}_{\mathrm{I}} 3$, indique un apport à Bâle supérieur à 100 ooo tonnes, soil $\mathbf{1} 50$ pour soo de plus qu'il y a quatre ans. Une fois la régularisation du lac de Constanci assurée, ce trafic, qui se borne à 200 jours en aval de Bâle, sera assuré pour la majeure partie de l'année, si ce n'ost pour l'annéc entière. Ge grand centre commercial scla alors en communicalion à peu près constante avec l'Oećan, ol l'on ne verra plus se reproduire des arrêts prolongés des approvisionnements en Allemagne, comme le fail s'est passí au cours de la crise balkanique, où x 500 wagons de blé, achetés par la Confédération, fur»nt retenus deux mois aux entrepôts de Mannheim, sous prétexte de manque de mátériel roulant. Cette masse énorme de farine n'aturait-elle pas été peut-être la bienvenue pour l'armée allemande en cas de conflit occidental européen ?

Notre confrère demande avec raison que l'article visé plus haut soit modifié et remplacé par la rédaction suivante : 
" les usines hydrauliques el aulres ouvages établis sur lis cours d'cau seront construits et cxploités de manière à assurer à la fois te maintien de la navigabilité, tolle qu'clle ćlait au moment de la construction, et le développement fulur de la navigation. Le concessionnaire de l'usine ou de l'ommage hydraulipue doil pourvoir, à ses frais, aux installations el ì l'amenéc de l'eau permetlant, à dive d'expert, lo passage régulier cl constant des bateaux du tonnage maximum correspondant a l'état génćral du chenal navigable avant la construction. "

Si, par contre, la concession impose à l'usine des obligalions plus étendues en faveur de la navigation, ou hors de proportion aux ressources du propriétaire, il y aura licu à dedonmagoments éventuels.

I a requêle est aussi modérée que justiliée, et tout amoindrissement de ces droils du public seraient un sacrifice incxcusable ef ploin de graves conséquences pour l'avenir de la navigation suisse.

\section{IRIX DÉCERNÉS PAR LACADÉMIE IEES SCIENGES}

Dans sa séance publique du 55 décombre 5913 , l'Académie des Sciences a remis aux lauréats de ses divers concours anmucls les prix qu'elle leur a décernés. Le bullelin correspondant des Comples Rendus conlient les rapports des commissaires de lous ces concours. Nous en extrayons les suivanls, qui so rapportent à des prix décernés dans la Section de Physique, eclle qui inléresse plus spécialement nos leclrurs. Ils concerncol d'alleurs des récompenses si justement décernées à des ingénicurs bien comus de nos lecteurs qui applaudiront avec nous à ces distinctions.

Le prix Héberl, d'une valcur de mille francs, est décerné à M. Swrnoedurw, professeur d'Electrotechnique à la Faculté des Scicnces de l'Université de Lille, qui s'est fait connaître dos physiciens et des technologistes par de nombreux traraux de Physique pure et appliquée.

Parmi Jes premicrs, il convient de signalor particulic̀rement ses études sur les potentiels explosifs. Dès ${ }^{8}{ }_{9} 6$, M. lo profosseur swingedarw a établi qu'il y a lieu do distinguer cutre le polcnliel explosif proprement dit, celui qui correspond à une chargo lente, cl celui qui caractérise les explosions brusques résultant, par exemple, de la décharge d'inlucteurs ou de condensateurs. Ce polentiel explosif dynamique peut, suivant que les électrodes sont ou non fréquemment polies, soumises on non à l'action de lumière ultraviololle provenant d'úlincelles voisines, ete, varicr de la moilié au friple de la valeur normale (potentiel explosil stalique).

Au mènc groupe de sujels se rapportent. les recherches de M. Swingedauw sur les décharges dans les excitateurs hortriens, les décharges dérivées, latérales, ete.

Mais c'est sturtoul dans los recherches rolatives aux diffciles problemes que souleve l'Flectrotechnique moderne que s'est spécialisé M. Swingedauw, depuis que, par ses fonclions de directem de l'Inslitut électrolechnique de l'Universilé de lille, qu'il a fondé dans des condilions délicates el bientol amené à rivaliser avec les établissements similaires les plus farorisés, il a dû orienler son aclivité dans une voie nouvelle.

Il s'est plus spécialement attaqué aux problèmes si complexes que soulève l'étude des dynamos à courant continu : aulo-cxcilation, réactions d'induit, commulation, pertes par Iỵstérésis et par courants de Foucanlt, rendement, etc. $\Lambda$ chacune de ces queslions, 11 . Swingedauw a su apporter des contributions originales. Ses recherches ont fait l'objet de Notes nombreuses insérées dans les Comples rendus de l'Académie des Sciences, de Mémoires publiées dans le Journal de Physique, le Bulletin de la Société internationale des Eleclriciens, l'Eclairage électrique, les Mémoires de l'Universili de Lille, de la Société industrielle du Nord, etc.

En dernicr lieu et tout récemment, M. Swingedauw a conmencé la publication de son Cours d'Electrolechnique génirale el appliquée. Lc premier volume, relatif à la machine dynamo à courant continu, publié avec le concours de deux de ses élèves les plus distingués, MM. Nègre et Beauvais, lui a fourni l'occasion de rassembler sous unc forme didaclique les principaux objets de ses recherches favorites, el d'exposer dans leur ensemble les questions qu'il a approfondies et dont il a contribué à éclaircir les solutions.

Le prix Gaston Planté, d'une valeur de deux mille francs, est décerné à M. R.-V. Picov, ingénieur des Arls ct Manufactures.

Les travaux de M. Picou comptent parmi ccux qui ont le plus contribué aux progrès accomplis depuis 25 ans dans le domaine de l'industrie électrique. Ses ouvrages sur les générateurs et moteurs, sur les canalisations et les réseaux ont rendu aux électriciens les plus grands services, et les méthodes qu'il a instituées pour le calcul des dynamos et la prédélermination de leur fonctionnement sont aujourd'hui classiques. Dans un autre ordre d’idées, M. Picou a singulièrement facilité l'élude, si nécessaire, des qualités magnétiques des fors et aciers en créant un perméamètre qui, par son principe même, élimine l'influence des joints ; aussi cet instrument est-il d'un usage courant dans les forges et ateliers de construction.

D'autres questions, non moins importantes, ont été heureusement résolues par M. Picou : dès I888, par l'application judicieuse des pôles auxiliaires, dont l'idée première est due à M. Mengès, il améliorait considérablement la commulation, à cette époque souvent médiocre, des machines électriques ct augmonlait ainsi notablement leur capacilé d'utilisation.

Plus tard, il imagina, pour la régulation des groupes électrogènes à vapeur, un disposilif très remarquable dans lequel l'admission, au lieu d'être fonction de la seule vitesse, dépend à la fois de la tension et du courant, c'est-à-dire des deux facteurs de la puissance électrique. L'admission de la vapeur peut dès lors suivre, indépendamment de la vitessc el de la tension, les variations de la charge, et ainsi sont évilés les ralentissements ou accélérations qui, avec les régulateurs ordinaires, constiment l'unique moyen de faire varier le couple moleur, obligcant ainsi à effectuer à la main, par la manoeuvre d'un rhéostal, le réglage de la tension.

Signalons encore une étude très approfondie des efforts magnétiques souvent énormes qui peuvent naître dans les machines puissantes, et des électro-aimanls à longue course pour la construction desquels M. Picou a donné une solution nouvelle, diflérente de celle déjà trouvée par M. Gućnée, ct dont le principe est le même que pour l'électromètre à cadranś ; ces appareils sont aujourd'hui cxtrêmement répandus dans les usines.

Les dynamos miltipolaires sont fréquemment le siège de courants de circulation provoqués par de légères dissymétrics el qui, dans les machines à grand débit, c'est-à-dire peu résistantes, peuvent acquérir des valeur énormes. On n'avait pas réussi, jusqu'à ce jour, à les atténuer suffisamment poù? pouvoir aborder la construction de très fortes machines à ćlectrolyse. Tout récemment, M. Picou a rósolu ce problème 